\title{
PGK1 Promotes NSCLC Progression by Enhancing Aerobic Glycolysis and Activating AKT/ERK Signaling Pathway
}

\section{Yongqin Tang}

Chuzhou Hospital Affiliated to Anhui Medical University

\section{Mei Li}

Chuzhou Integrated Traditional Chinese and Western Medicine Hospital

Jinxuan Hei

Chuzhou Hospital Affiliated to Anhui Medical University

\section{Likun Ma}

The First Affiliated Hospital of USTC: Anhui Provincial Hospital

Jianyuan Pan ( $\nabla$ doc_jianyuanpan@163.com )

The First Affiliated Hospital of University of Science and Technology of China

\section{Research}

Keywords: metabolic reprogramming, aerobic glycolysis, Warburg Effect, hypoxia

Posted Date: November 9th, 2021

DOI: https://doi.org/10.21203/rs.3.rs-1046059/v1

License: (c) (i) This work is licensed under a Creative Commons Attribution 4.0 International License. Read Full License 


\section{Abstract}

Metabolic reprogramming, especially aerobic glycolysis is considered a hallmark of cancer, and is becoming a novel target for cancer therapy. Phosphoglycerate kinase I (PGK1) which is an important enzyme generating the first ATP in glycolysis pathway was already shown can promote some types of cancer development and progression, however, the role of PGK1 in lung cancer development is less reported. The aim of this study was to explore the mechanism of PGK1 in promoting non-small cell lung cancer (NSCLC) development and progression. Gene overexpression or silencing, scratch assay, Transwell assay, western blot, immunohistochemistry, chromatin immunoprecipitation, real-time quantitative RT-PCR, MTT cell viability assay and mouse xenograft, glucose uptake, lactate secretion, ATP production and Extracellular Acidification Rate (ECAR) seahorse assay were performed to investigate the biological function and the underlying mechanism of PGK1 in NSCLC development. Our study found that PGK1 was high expressed in non-small cell lung cancer tissues, its expression is positive correlated with tumor grade and clinical stage and negative correlated with patients' overall survival. Importantly, its expression was also associated with clinicopathological characteristics of lung cancer patients. Overexpression PGK1 could not only promote lung epithelial cell and tumor cell migration and proliferation in vitro, but also increase the tumor growth in vivo. Mechanically, PGK1 promotes NSCLC development and progression by activating AKT/ERK signaling pathway and altering aerobic glycolysis pathway. Meanwhile PGK1 was HIF1a downstream target gene and played an essential role in hypoxia-induced shift of glycolysis. In addition, the PGK1 expression was positive correlated with HIF1a expression in NSCLC tissues. Therefore, we concluded that PGK1 could be a biomarker for NSCLC diagnosis and outcome evaluation, and targeting PGK1 should be an innovative strategy for NSCLC therapy in the future.

\section{Introduction}

Lung cancer is the most commonly diagnosed cancer and accounts for $18.4 \%$ of cancer-related death worldwide which is the leading cause of cancer death both in men and women[1]. Lung tumors can be divided into two main histopathological categories: small-cell lung cancer (SCLC) and non-small-cell lung cancer (NSCLC). About $80 \%$ of lung cancer cases are NSCLC, which is composed of three major pathological subtypes: adenocarcinoma, squamous cell carcinoma and large-cell carcinoma[2]. Excision, chemotherapy and radiotherapy are the three major therapeutic strategies to treat lung cancer. Although there have been advances in targeted therapies and immunotherapies, the 5-year survival rate remains only $15 \%[3]$. Therefore, how to improve the clinical outcomes of the NSCLC patients is remain a challenge.

Metabolism reprogramming has been considered as a hallmark of cancer. Tumor cell prefer to process glycolysis to generate ATPs even in the presence of oxygen (Warburg Effect)[4]. Because of the aerobic glycolysis, tumor cells consume more glycose compared to the normal cells, and more lactate is secreted as a metabolite nearby the tumor cells, supporting an acidic microenvironment which can promotes tumor cells growth and metastasis[5]. Thus, targeting Warburg Effect has become a prevalent therapeutic strategy, and mounting studies are focusing on its potential to treat cancer. 
Hypoxia is a common reason to cause metabolic shift and acidic microenvironment in cancer[6]. HIF1a is the main transcriptional regulator response to hypoxia[7] and involve in kinds of biological processes in cancer, include angiogenesis[8], cell invasion [9] and glycolysis[10]. Some of the rate limiting enzymes of the glycolysis are considered as targets for inhibiting Warburg Effect, such as HK2 and GAPDH[11-15]. It was also reported these genes could be regulated by HIF1a[16, 17]. PGK1 is one of the central enzymes in the glycolysis pathway, which catalyzes the conversion of 1,3-diphosphoglycerate to 3-phosphoglycerate and generates a molecule of ATP and it has been shown dysregulated in different kinds of tumors. In certain type of tumor, PGK1 was considered as an anti-tumor factor, Lay AJ[18] found that extracellular PGK1, which is secreted by tumor cells, can inhibit tumorigenesis and angiogenesis as a disulphide reductase. However, more studies showed that PGK1 was an oncogene in most of tumors, for example: colon cancer[19], oral cancer[20], endometrial cancer[21] and its expression was negatively correlated patients' survival. It was also known that PGK1 promotes breast cancer development by regulating the HIF1a-Mediated EMT[22]. Nevertheless, what is the function of PGK1 in lung cancer development and whether HIF1a plays a role here is poorly understand.

In our paper, we uncovered a key function of PGK1 as an oncogene in lung cancer development. Mechanistically, we showed that PGK1 could promote NSCLC cells proliferation and migration capacities via altering metabolism pathway and activating AKT/ERK signaling pathway. Meanwhile, $P G K 1$ is a downstream gene of HIF1a and play an essential role in hypoxia-induced glycolysis alteration. Thus, our study indicate that PGK1 could be a potential survival biomarker of NSCLC patients, provide a new link between metabolism reprogramming and the malignant phenotype, which can be an innovative target therapy for lung cancer.

\section{Results}

PGK1 is high expressed in lung cancer tissues and its expression level is positive correlated with lung cancer grade and stage and negative correlated with patients' overall survival. We first performed the immunohistochemistry $(\mathrm{IHC})$ staining of PGK1 in non-small cell lung cancer patients' tissues involve in different types, grades and clinical stages to determine whether irregular expression of PGK1 could been observed in NSCLC patients (Fig. 1). We found that PGK1 was higher expressed in grade 3 and stage III+IV lung cancer tissues of both adenocarcinoma and squamous cell carcinoma (Fig. 1, A-C). This can be confirmed by both in terms of intensity and percentage of positive cells. Whereas in grade 2 and stage II, especially in grade1 and stage I tumors, much fewer cells expressed PGK1, and at substantially lower levels (Fig. 1, A-C). The survival analysis showed that the overall survival rate of high expression PGK1 patients was significantly reduced compared with those with low PGK1 expression (Fig. 2, A). Together, these findings support the hypothesis that upregulation of PGK1 may play a role in promoting lung cancer progression and PGK1 maybe a potential biomarker for NSCLC patients' outcome.

PGK1 expression is correlated with clinicopathological characteristics in lung cancer patients. We further measured the PGK1 expression in all the 90 patients' tissues by IHC. Semi-quantitative analysis showed that the PGK1 expression was significantly higher in moderately and poorly differentiated NCSLC tissues 
compared with that in well-differentiated NSCLC tissue samples (Table 1). Meanwhile, PGK1 expression in NSCLC tissue samples with lymph node metastasis was also demonstrated to be significantly higher compared with that in NSCLC specimens without lymph node metastasis (Table 1). The relationship between PGK1 expression and the clinicopathological features of patients with NSCLC was further examined. PGK1 expression was found to significantly associate with tumor differentiation, clinical stage, T stage and lymph node metastasis, but not with age and sex (Table 1).

Table 1

Association between clinicopathological features in patient with lung cancer and PGK1 expression $(n=90)$.

\begin{tabular}{|c|c|c|c|c|}
\hline \multicolumn{5}{|l|}{ PGK1 expression } \\
\hline Clinicopathological features & NO. of cases & $\operatorname{High}(n)$ & Low(n) & P-value \\
\hline Sex & & & & 0.251 \\
\hline Male & 55 & 35 & 20 & \\
\hline Female & 35 & 18 & 17 & \\
\hline Age & & & & 0.787 \\
\hline$\geq 55$ & 52 & 30 & 22 & \\
\hline$<55$ & 38 & 23 & 15 & \\
\hline Differentiation & & & & 0.001 \\
\hline Well & 19 & 5 & 14 & \\
\hline Moderate +poor & 71 & 48 & 23 & \\
\hline T stage & & & & 0.024 \\
\hline $\mathrm{T} 1+\mathrm{T} 2$ & 61 & 31 & 30 & \\
\hline $\mathrm{T} 3+\mathrm{T} 4$ & 29 & 22 & 7 & \\
\hline Clinical stage & & & & 0.042 \\
\hline I-II & 57 & 29 & 28 & \\
\hline III-IV & 33 & 24 & 9 & \\
\hline LN metastasis & & & & 0.003 \\
\hline N- & 49 & 22 & 27 & \\
\hline $\mathrm{N}+$ & 41 & 31 & 10 & \\
\hline
\end{tabular}


PGK1 plays a role in enhancing cell migration capacity. To further analyze whether PGK1 play a role in cancer development in vitro, we generated PGK1 overexpression (OE) stable cell line by lentivirus-driven system in B2B(BEAS-2B) and A549 cell lines. From the wound-healing assay we can found that when overexpress PGK1, the migration capacity was significantly enhanced in both B2B and A549 cell line compared with control (Fig. 2, B-D). The Boyden chamber-based migration assay also showed that the higher migration capacity was observed in PGK1 overexpressed B2B and A549 cell lines (Fig. 2, E and F). We then generated the PGK1 knockdown (KD) cell lines by lentivirus-driven shRNA-mediated silencing both in B2B and A549 cells. We chose two different shRNAs of PGK1 to avoid the off-target effect. The migration capacity was measured by trans-well assay again (Fig. 2, G), and it showed significantly reduced in both B2B and A549 cell lines when PGK1 expression was silenced by the two different shRNAs (Fig. 2, H). Thus, PGK1 could promote migration in both lung epithelia and cancer cells.

PGK1 promotes cell proliferation both in vitro and in vivo. We next examined whether PGK1 could promote cell proliferation. Immunofluorescence (IF) assay for Ki-67 was performed in control and PGK1 $\mathrm{OE}$ cell lines, along with the $\mathrm{Ki}-67$ index analysis, the data showed that $\mathrm{Ki}-67$ positive cells were significantly increased in PGK1 overexpressed B2B and A549 cell lines compared with each control (Fig. 3, A-D). Cell viability and proliferation assay (MTT) also been performed and the results showed that when PGK1 was overexpressed in B2B and A549 cell lines, the proliferation ability was significantly increased compared with each control group (Fig. 3, E and F). While PGK1 knockdown had the opposite effects. The cell proliferation capacity was significantly weakened when PGK1 was knockdown by the two shRNAs (Fig. 3, E and F). To investigate the effect of PGK1 on tumor growth in vivo, PGK1 overexpression, PGK1 knockdown and control A549 cells were injected subcutaneously in BALB/c nude mice and kept growth for 6 weeks. The tumor volume was measured every week. The results showed that the tumor volume of mice transplanted with PGK1 overexpressed A549 cells was significantly larger than the control, while the volume was significantly smaller in transplanted with both shPGK1-1 and shPGK1-2 A549 cells compared with control (Fig. 3, G and H). Thus, these findings demonstrated that PGK1 promotes NSCLC cell proliferation both in vitro and in vivo.

\section{Dysregulation of PGK1 expression affects aerobic glycolysis in lung epithelial and tumor cells. The} glycolysis pathway is the main metabolic pathway for tumor cells to generate ATPs, and the PGK1 is a major enzyme in glycolysis pathway. To explore whether dysregulate PGK1 could affect the glycolysis capacity in B2B and NSCLC cells, The glucose uptake assay, lactate production and ECAR (Extracellular acidification rate) assay were performed. We found that overexpression PGK1 in B2B cell would significantly increase glucose uptake, lactate and ATP production (Fig. 4, A-C), meanwhile the seahorse assay also showed that both the glycolysis capacity and glycolysis reserve were enhanced in PGK1 OE cell lines (Fig. 4, D). Whereas knockdown PGK1 showed the opposite effect (Fig. 4, A-C and E). In NSCLC cells, A549 cell lines, the glucose uptake, lactate and ATP production, glycolysis capacity and glycolysis reverse were significantly increased when PGK1 was overexpressed (Fig. 4, F-I), while these features were significantly decreased when PGK1 was knockdown by different shRNAs (Fig. 4, F-H and J). Together, 
these date improved that dysregulate PGK1 would affect the glycolysis pathway of both B2B and NSCLC cells.

PGK1 promotes NSCLC cell proliferation through the AKT/ERK signaling pathway. Paper[23] showed that PGK1 could activate AKT signaling pathway in oral squamous cell carcinoma. Therefore, we want to investigate whether AKT signaling pathway would be activated by PGK1 in B2B and A549 cells. The activated AKT was measured in these cell lines. PGK1 overexpression was found to significantly increase p-AKT phosphorylation in both B2B and A549 cell lines (Fig. 5, A and C), while PKG1 knockdown by the two different shRNAs would reduce AKT phosphorylation obviously (Fig. 5, B and D). MAPK families play an important role in cell proliferation, differentiation, transformation, apoptosis and some other cellular programs[24]. Therefore, we further checked the MAPK families' cascades in both B2B and A549 cell lines when PGK1 was dysregulated. The results showed that the $p$-ERK phosphorylation was significantly increased when PGK1 was overexpressed in both B2B and A549 cells (Fig. 5, A and C), while PGK1 knockdown was found to reduce p-ERK phosphorylation in these two cell lines (Fig. 5, B and D). Meanwhile the activated JNK/SAPK and p-38 were unchanged in neither PGK1 overexpression nor PGK1 knockdown B2B and A549 cells (Fig. 5, A-D). These findings suggested that PGK1 might promote NSCLC progression by activating the AKT/ERK signaling pathway.

\section{PGK1 can be directly regulated by HIF1a and plays an important role in HIF1a-induced alteration of} glycolysis pathway. HIF1a is a central regulator to promote cancer cell survival and progression in solid tumors by inducing glycolysis[25]. PGK1 is a target gene of HIF1a, in our study, we found that both PGK1 protein and RNA level were significantly increased when the A549 cells were treated in hypoxia condition (Fig. 6, A and B). We performed HIF1a Chip-qPCR assay in A549 cell line to confirm whether PGK1 was HIF1 a target gene in NSCLC (Fig. 6, C). The IgG Chip was performed as negative control. The primers in the promoter region of $P G K 1$ and VEGFa were designed, the VEFGa was considered as positive control of HIF1a-Chip[26], whereas primers from 3'region of $F G F 5$ gene was designed as a negative control of HIF1a-Chip, the q-PCR date showed that the PGK1 and VEGFa enrichments were significantly increased in hypoxia treated A549 cells compared with untreated cells, while the FGF5 enrichment almost didn't change(Fig. 6, C). Thus, PGK1 was improved to be one of the HIF1 a downstream target gene. The seahorse assay was performed again and the data showed that when A549 cells were treated in hypoxia for 24h, the glycolysis capacity and reverse were significantly increased, while PGK1 knockdown could rescue the hypoxia-induced increased glycolysis (Fig. 6, D and E). Meanwhile, the results of glucose uptake lactate secretion and ATP production assays all showed increased in hypoxia treated A549 cells and decreased in PGK1 KD counterparts (Fig. 6, F-H). So together the findings we concluded that PGK1 is a downstream target gene of HIF1a and plays an important role in hypoxia-induced alteration of glycolysis pathway in NSCLC cells.

HIF1a levels positively correlate with PGK1 levels in lung cancer patients. To analyze whether there is a correlation between PGK1 and HIF1a expression level in lung cancer patients, we performed the IHC of 
HIF1a in patients' samples (Fig. 7, A). HIF1a was almost undetectable in normal lung tissue, while was significantly upregulated in both lung adenocarcinoma and lung squamous cell carcinoma (Fig. 7, A and B). Importantly, the HIF1a expression in the tumors was also positively correlate with the tumor clinical stages (Fig. 7, B). The analysis of staining intensity of PGK1 and HIF1a supporting a positive link between PGK1 and HIF1a expression in lung cancer patients (Fig. 7, C).

\section{Discussion}

Mounting studies focus on investigating the relationship between tumor and metabolism recently, especially the glucose metabolism. Abnormal glucose metabolism is a distinguishing feature of tumor metabolism, thus, targeting aerobic glycolysis is becoming a new strategy for treating cancer[27]. There were some paper reported that some of the rate limiting enzymes of glycolysis were involve in NSCLC development. For example, Patra et al. [28]showed that HK II (Hexokinase II) is required for lung cancer development in mouse models. Minchenko et al.[29] also observed PFK (Phosphofructokinase) mRNA is upregulated in human lung cancer tissues and A549 cells compared with normal tissues, and could be induced by hypoxia. In addition, Xie et al.[30] found that LDHA (Lactate dehydrogenase) play an essential role in lung cancer cell survival and proliferation, and inhibit LDHA can be a potential therapeutic target for NSCLC. According our study, we confirmed that PGK1 promote lung cancer development via altering metabolic pathway, supporting a new link between glycolytic pathway and lung cancer development.

AKT signaling pathway has been reported to regulate cell proliferation[31]. Aberrant activation of AKT is associated with malignancy[32]. It has been showed that the amplification of AKT promotes the tumorigenesis and progression in human gastric adenocarcinoma[33], ovarian and breast carcinoma[34] and human pancreatic carcinoma[35]. Amplification of phosphatidylinositide-3-kinase (PI3K) and deletion of PTEN, a PI3K antagonist, are the common upstream activator of AKT; meanwhile, AKT is also a critical downstream effector of some oncogenes, for example Bcr-Abl, Her2/neu, and Ras. Some paper also reported that AKT stimulates aerobic glycolysis that correlates with a more aggressive malignancy in human glioblastoma[36]. Some of the glycolytic enzymes, including GLUT1 and GLUT4[37], also can be activated by increasing of AKT signaling. MAPK families include three main members: named ERK, CJun-N-terminal kinase/ stress-activated protein kinase (JNK/SAPK) and P-38 kinase, which are the three main chain of proteins in the cell that transduce signals from the receptors on the cell surface to the DNA in the nucleus of the cell. It is well known that MAPK signal pathway plays an essential role in the regulation of cell proliferation in mammalian cells[24], especially the ERK signaling pathway is reported implicated in tumorigenesis[38]. Our present study date suggests that the expression level of PGK1 was positively correlated with the phosphorylation level of AKT and ERK in both B2B and A549 cells. These data supports a new chain of glycolytic enzyme (PGK1) -phosphorylation AKT - phosphorylation ERK1/2 - tumorigenesis, which may explore a new sight of target therapy for lung cancer. However, the more detailed mechanism of how PGK1 affect AKT and ERK phosphorylation and what the downstream effect of P-ERK is still elusive and need to be further investigated. 
Most human solid tumors are subjected to hypoxia in the way of acute or chronic because of aberrant vascularization and a poor oxygen supply[39]. Hypoxia-inducible factor a-1 (HIF1a) is the main response of hypoxia[40]. Most tumor cell will change its behavior via HIF-dependent pathway, leading to cancer progression, angiogenesis, metastasis and radiation and drug resistance[41]. One of the most important and canonical pathway is the alteration of glycolysis, which has been thoroughly studied. It has been reported that HIF1 a was overexpressed in NSCLC tissue compared with normal tissue and was related to the enhancing of angiogenesis and with poor prognosis[42]. Our date also found that HIF1a was upregulated in NSCLC tissue and has a positive correlation with tumor clinical stage and PGK1 expression. Our data confirmed PGK1 is a downstream target gene of HIF1a. These date supply an additional supplementary evidence to confirm the glycolytic pathway changed via HIF1a-dependent way during NSCLC development.

\section{Conclusion}

Our date suggests that PGK1 is a potential biomarker for NSCLC patients' diagnosis and survival evaluation, and PGK1 may promote NSCLC progression by altering tumor cells glycolytic pathway and activating AKT/ERK signaling pathway. PGK1 is HIF1a downstream target gene and plays an important role in hypoxia-induced alternation of glycolytic pathway.

\section{Material And Methods}

\section{Patients and tissue samples}

We selected 90 cancer tissue samples from NSCLC inpatients (Age range, 39-76 years; sex, 55 males and 35 females) and 20 matched cancer adjacent tissue samples in total from The First Affiliated Hospital of University of Science and Technology of China (USTC). All tissue samples taken from the patients were approved by the ethical review of Committee of The First Affiliated Hospital of USTC. All samples were performed immunohistochemistry staining for PGK1 and HIF1a. All patients received radical surgery; the patients who received any form of pre-surgical adjuvant therapy were excluded. The clinical and pathological characteristics (sex, age, differentiation, $T$ stage, clinical stage and lymph node metastasis) of NSCLC were established in accordance with the criteria of The Eighth Edition Lung Cancer Stage Classification[43].

\section{Cell culture, generation of the stable cell line}

BEAS-2B (B2B), A549, HEK293T cell lines were purchased from American Type Culture Collection. HEK293T cells were cultured in DMEM medium supplemented with $10 \%$ fetal bovine serum (FBS) and $1 \%$ penicillin/streptomycin/glutamine (PSG). B2B and A549 cells were cultured in Roswell Park Memorial Institute 1640 supplemented with 10\% FBS and PSG. For generate the PGK1 overexpression (OE) stable cell line, PGK1 cDNA of human was synthesized and cloned in p-Lenti-EF1a-Backbone plasmid. The primers were listed in Table S1. The restriction enzyme sites are BamHI and Xbal. For generating PGK1 knockdown (KD) stable cell line, we chose two different shRNAs (constructed in PLKO plasmid) which 
were purchased from Thermo Fisher. $0.5 \times 10^{6}$ HEK293T cells were seeded in 6-well plate and transfected with 2ug of control, PGK1-overexpression and shRNAs plasmid along with the packaging plasmid using the Fu-GENE transfect reagent. $72 \mathrm{~h}$ after transfection, harvested the viral supernatant, then transduced into B2B and A549 cell lines. 48h after transduction, cells were selected with $10 \mathrm{ug} / \mathrm{ml}$ puromycin for two passages and were maintain cultured in normal medium with $2 \mathrm{ug} / \mathrm{ml}$ puromycin.

\section{Immunohistochemistry (IHC) and immunofluorescence staining}

For immunohistochemistry staining on paraffin sections, slides first were heated at $55^{\circ} \mathrm{C}$ for $10 \mathrm{~min}$ and then merged into xylene for $5 \mathrm{~min}$, followed by consecutive dewaxing steps ( $2 \times$ xylene, $100 \%$ ethanol, $75 \%$ ethanol, $50 \%$ ethanol, 5 min each). Then boiled the slides in $10 \mathrm{mM}$ citrate buffer by microwave for $10 \mathrm{~min}$ for the purpose of antigen retrieval, cooling the slides in room temperature. The subsequent processes were performed with the VECTASTAIN Universal Quick HRP Kit (PK-7800; Vector Laboratories) following the manufacturer's instructions. DAB Peroxidase (HRP) Substrate Kit (SK-4100; Vector Laboratories) was used for staining developing. Images were acquired by an Axio Imager A2 microscope.

For immunofluorescence staining of culture cells, 100,000 cells were seeded on coverslips in 24-well plates. Next, cells were washed with PBS and fixed by 4\% PFA for 10 min. Then cells were blocked with $5 \%$ BSA, $0.2 \%$ Triton X-100 in PBS for 1 hour and incubated with primary antibody in blocking buffer at $4^{\circ} \mathrm{C}$ overnight. Next day, cells were incubated with secondary antibody for 2 hours in room temperature in dark, followed by incubating with DAPI for $20 \mathrm{~min}$, and then coverslips were washed and mounted in antifade mounting medium. The fluorescence images were acquired by using CONFOCAL microscope with a $25 \times$ or $63 \times$ objective and analyzed with ImageJ Software.

\section{Quantification of immunoreactivity}

For semi-quantitative analysis of immunoreactivity of PGK1 and HIF1a, we used H-score in this study. We randomly selected 10 different tumor fields in a×400 magnification sight, at least 100 cells in each sight, and the $\mathrm{H}$-score was generated by adding the percentage of strongly stained( $3 \times$ ), the percentage of moderately stained $(2 x) \square$ the weakly stained( $1 \times)$, giving a range of $0-300$. Two of the authors obtained the score independently, and the authors did not know any clinical parameters of patients. The differences of interobserver were less than $5 \%$ in this study, and the mean of the two values was used. In the case of the percentage of PGK1 immunostaining intensity in moderately + strongly was more than $50 \%$, we defined it as high expression. Ki-67 labeling index was used to describe the Ki-67 immunoreactivity. Image J was used to quantify the Ki-67 positive cells based on fluorescent nuclear signal intensity and manually tuned to obtain the accurate results. At least 1000 cells in 10 random views were selected to analyze.

\section{Scratch wound assay and Boyden chamber migration assay}

Both B2B, A549 with control, PGK1 OE cells were seeded in 6-well plate. When cells reaching $100 \%$ confluence, scratches were made by 10 ul pipette tips, washed the cell by PBS 3 times, then culture the cells in the medium without FCS, images were acquired after scratching, as well as $24 \mathrm{~h}$ and $48 \mathrm{~h}$ later. For 
Boyden chamber assay, the inserts were placed on 24-well plate contained normal culture medium. 120,000 B2B and 50,000 A549 cells with control, PGK1 OE and PGK1 KD were seeded on the top of inserts in the medium without FCS. 8 hours later (for A549 cells, 24h later), the insert membranes were fixed by $4 \%$ PFA for $10 \mathrm{~min}$ and washed by PBS 3 times. The upper surface of the membranes was first cleaned by wiping with a cotton swab. Then the membranes were stained with crystal violet for $10 \mathrm{~min}$. Then washed the membranes by PBS 3 times, the membranes were cut and mounted on the slides. Images were acquired from random area of the membrane by 20x objective microscope, and the cells which migrated to the lower surface of the membrane were quantified by ImageJ. For each migration assay, a minimum of three independent experiments were performed.

\section{RNA isolation and q-PCR}

RNA was isolated by using TRIzol RNA Isolating Reagent (Invitrogen). For quantification PCR analysis, cDNA was synthesized with the High-Capacity cDNA Reverse Transcription Kit (Applied Biosystems), and Q-PCR was performed by using the SYBR Green PCR master mix. Cycle numbers were normalized to these of $\beta$-actin. $q$-PCR primers used in this research can be found in Table S1.

\section{Western Blotting}

Cells were cultured in normal medium, when cells reached to $70 \%-80 \%$ confluence; cells were washed and lysed in radioimmunoprecipitation assay (RIPA) buffer supplemented with proteinase inhibitor and phosphorylase inhibitor. The lysate was harvest by scratching. All the experiments were performed on ice. The concentration of protein was measured by pierce BCA protein assay kit (Thermo Fisher, Cat\#:23227). The same amount of proteins were mixed with $5 X$ Leammli sample buffer and boiled at $95^{\circ} \mathrm{C}$ for 10 min. Protein was loaded on the SDS-PAGE gel and separated by electrophoresis, transferred onto PVDF membrane. Membranes were stained by ponceau for 1 min and then blocked by blocking buffer (PBS+0.1\% Tween-20+5\% BSA) in room temperature for 1 hour. Then the membranes were incubated with primary antibody at $4{ }^{\circ} \mathrm{C}$ overnight. The detail of primary antibodies used in this research were presented in Table S2. Then the membranes were incubated with appropriate secondary antibody in room temperature for 1 hour. Immunoreactive bands were observed by chemiluminescence. Three independent experiments were performed.

\section{Cell proliferation assay (MTT)}

The MTT assay was performed follow by the protocol from Promocell (Cat\#: PK-CA707-30006). 3000 cells were seeded in 96-well plates with 100ul medium. When the cells were attached to the plate, added 10ul MTT solution to each well and placed the plates in the culture incubator for 4 hours. 200ul DMSO was added to each well and pipette up and down several times to dissolve the formazan salt. Measured the absorbance signal on a spectrophotometer at $570 \mathrm{~nm}$, measured background absorbance at $630 \mathrm{~nm}$. To obtain normalized absorbance value the substrate background absorbance from signal absorbance $\mathrm{OD}_{570}-\mathrm{OD}_{630}$. Three duplicated in each cell line group, and three independent experiments were performed in this assay.

\section{Animal experiment}


BALB/c nu/nu mice were purchased from Cyagen Biosciences Company, Soochow city, China, and bred under pathogen-free conditions. For subcutaneous transplantation, $10^{7}$ control, PGK1 OE and PGK1 shRNA-silencing A549 cells were subcutaneously injected into the flanks of BALB/c nu/nu mice. The tumor size was measured every week. The tumor volume was calculated by the following formula: volume $=\left(\right.$ longest diameter $\times$ shortest diameter $\left.{ }^{2}\right) / 2$. After 7 weeks, the mice were sacrificed, and the tumors were frozen in liquid nitrogen for further study. Unexpected died mice were excluded from analysis.

\section{Glucose uptake, lactate and ATP production assay and seahorse assay}

Glucose Uptake Colorimetric Assay Kit (Biovision, Cat\#: K676-100), Lactate Assay Kit (Cell Biolabs, Cat\#: MET-5012) and ATP colorimetric Assay Kit (Biovision, Cat\#: K959) were used to measure glucose uptake and the lactate, ATP production according to the manufacturer's instructions. The extracellular acidification rate (ECAR) was measured using the seahorse XFe 24 Extracellular Flux Analyzer according to the manufacturer's instruction. ECAR were determined by using Seahorse XFe Glycolysis Stress Test Kit (Agilent, Cat\#: 103017-100). Briefly, 3×104 B2B cells and $4 \times 10^{4}$ A549 cells were seeded into a seahorse

XFe 24 cell culture microplate. After calibration and baseline measurements, glucose $(10 \mathrm{mM})$, the oxidative phosphorylation inhibitor oligomycin (1uM), and the glycolytic inhibitor 2-DG (50mM) were sequentially injected into each well at certain time points. Date was analyzed by seahorse XFe 24 Wave software. Four repeat wells in each cell line, and three independent assays were performed in these measurements.

\section{Chip and Chip-qPCR}

$10^{7} \mathrm{~A} 549$ cells treated in normoxia and hypoxia were fixed with $1 \%$ formaldehyde for $10 \mathrm{~min}$ at room temperature. $125 \mathrm{mM}$ glycine was added to quench the fixation. Cells were suspended in cold PBS in the concentration of $5 \times 10^{6} / \mathrm{ml}$. Added equal volume of $2 \times$ lysis buffer $(100 \mathrm{mM}$ Tris- $\mathrm{HCl}$ pH8, $300 \mathrm{mM} \mathrm{NaCl}$, $2 \%$ Triton- $100,10 \mathrm{mM} \mathrm{CaCl} 2$ ) for $10 \mathrm{~min}$ on ice with shaking. Next, nuclei were sheared by MNase $\left(7.8 \mathrm{U} / 5 \times 10^{6} \mathrm{cells}\right)$ for $15 \mathrm{~min}$ at $37^{\circ} \mathrm{C}$. Stop the reaction by adding $20 \mathrm{mM}$ EDTA on ice $30 \mathrm{~min}$. The extracts were pre-cleaned with $75 \mathrm{ul}$ protein $\mathrm{G}$ beads for $2 \mathrm{~h}$ in $4^{\circ} \mathrm{C}$, then incubated with $12.5 \mathrm{ug}$ HIF1a primary antibody and same amount of rabbit IgG respectively overnight at $4^{\circ} \mathrm{C}$ and binding to $75 \mathrm{ul}$ BSA-coated protein $\mathrm{G}$ beads. Immunoprecipitates were washed by following steps: twice with low salt buffer (0.1\%SDS, $1 \%$ Triton-100, 2mM EDTA, 20mM Tri-HCl pH7.5, $150 \mathrm{mM} \mathrm{NaCl}$ ), three times with high salt buffer (0.1\%SDS, $1 \%$ Triton-100, 2mM EDTA, $20 \mathrm{mM}$ Tri-HCl pH7.5, $500 \mathrm{mM} \mathrm{NaCl),} \mathrm{twice} \mathrm{with} \mathrm{LiCl} \mathrm{buffer}$ (10mM Tris-HCl pH7.5, 250mM LiCl, 1\% NP-40, 1\%Na-Doc, 20mM EDTA) and then eluted with Elution buffer ( $50 \mathrm{mM}$ Tris-HCl pH 8.0,10mM EDTA pH8.0, 1\%SDS). After treaded samples by adding $1 \mathrm{ul} \mathrm{RNase} \mathrm{A}$ $1 \mathrm{~h}$ at $37^{\circ} \mathrm{C}$ and $10 \mathrm{ul}$ Protein $\mathrm{K} 2 \mathrm{~h}$ at $37^{\circ} \mathrm{C}$, cross-linking was reversed by incubation at $65^{\circ} \mathrm{C}$ overnight. DNA was purified by using Qiagen mini elute PCR purification kit (Cat\#28004). Then concentration of immunoprecipitated DNA was measured by Qubit. The q-PCR was performed to analyze the enrichment. Chip-qPCR primers can be found in Table S1. 


\section{Statistical analysis}

All date was statistically analyzed by using SPSS 25.0 software. Independent sample $t$-test was used for comparison between two groups. $\chi^{2}$ test was used to estimate the association between PGK1 expression and the clinicopathological characteristics of patients with NSCLC. The Kaplan-Meier method and logrank test were used to compare the overall survival of patients. Data were presented as mean \pm SD. $\mathrm{P}<0.05$ was considered statistically significant.

\section{Declarations}

\section{Acknowledgements}

Not applicable.

\section{Conflict of interest statement}

The authors declare no competing financial interests.

\section{Author contributions}

Jianyuan Pan and Likun Ma developed the concept of this research. Yongqin Tang performed the experiments, analyzed the data and wrote the manuscript. Mei Li did the animal xenograft assay; Jinxuan Hei collected and analyzed the clinical samples and clinical data. All authors discussed the results and commented on the manuscript.

\section{Ethics statement}

The tissue samples from patients were approved by the ethical review committee of The First Affiliated Hospital of University of Science and Technology of China (USTC), Hefei, China. The study methodologies conformed to the standards set by the Declaration of Helsinki.

\section{Funding statement}

The author received no specific funding for this work.

\section{Data availability}

All data supporting the findings of this study are included in the manuscript and are available from the corresponding author on request.

\section{References}

1. Bray, F., et al., Global cancer statistics 2018: GLOBOCAN estimates of incidence and mortality worldwide for 36 cancers in 185 countries. CA Cancer J Clin, 2018. 68(6): p. 394-424. 
2. Dela Cruz, C.S., L.T. Tanoue, and R.A. Matthay, Lung cancer: epidemiology, etiology, and prevention. Clinics in chest medicine, 2011. 32(4): p. 605-644.

3. Siegel, R.L., K.D. Miller, and A. Jemal, Cancer statistics, 2019. CA Cancer J Clin, 2019. 69(1): p. 7-34.

4. Warburg, O., On the origin of cancer cells. Science, 1956. 123(3191): p. 309-14.

5. Boedtkjer, E. and S.F. Pedersen, The Acidic Tumor Microenvironment as a Driver of Cancer. Annu Rev Physiol, 2020. 82: p. 103-126.

6. Chiche, J., M.C. Brahimi-Horn, and J. Pouyssegur, Tumour hypoxia induces a metabolic shift causing acidosis: a common feature in cancer. J Cell Mol Med, 2010. 14(4): p. 771-94.

7. Wang, G.L., et al., Hypoxia-inducible factor 1 is a basic-helix-loop-helix-PAS heterodimer regulated by cellular $\mathrm{O} 2$ tension. Proceedings of the National Academy of Sciences of the United States of America, 1995. 92(12): p. 5510-5514.

8. Pugh, C.W. and P.J. Ratcliffe, Regulation of angiogenesis by hypoxia: role of the HIF system. Nature Medicine, 2003. 9(6): p. 677-684.

9. Fujimura, A., et al., Cyclin G2 promotes hypoxia-driven local invasion of glioblastoma by orchestrating cytoskeletal dynamics. Neoplasia (New York, N.Y.), 2013. 15(11): p. 1272-1281.

10. Kierans, S.J. and C.T. Taylor, Regulation of glycolysis by the hypoxia-inducible factor (HIF): implications for cellular physiology. J Physiol, 2021. 599(1): p. 23-37.

11. Zhong, X.Y., et al., CARM1 Methylates GAPDH to Regulate Glucose Metabolism and Is Suppressed in Liver Cancer. Cell Rep, 2018. 24(12): p. 3207-3223.

12. DeWaal, D., et al., Author Correction: Hexokinase-2 depletion inhibits glycolysis and induces oxidative phosphorylation in hepatocellular carcinoma and sensitizes to metformin. Nat Commun, 2018. 9(1): p. 2539.

13. Liberti, M.V., et al., A Predictive Model for Selective Targeting of the Warburg Effect through GAPDH Inhibition with a Natural Product. Cell Metab, 2017. 26(4): p. 648-659.e8.

14. Yun, J., et al., Vitamin C selectively kills KRAS and BRAF mutant colorectal cancer cells by targeting GAPDH. Science, 2015. 350(6266): p. 1391-6.

15. Wolf, A., et al., Hexokinase 2 is a key mediator of aerobic glycolysis and promotes tumor growth in human glioblastoma multiforme. J Exp Med, 2011. 208(2): p. 313-26.

16. Wyatt, E., et al., Regulation and cytoprotective role of hexokinase III. PLoS One, 2010. 5(11): p. e13823. 
17. Zhang, J.Y., et al., Critical protein GAPDH and its regulatory mechanisms in cancer cells. Cancer Biol Med, 2015. 12(1): p. 10-22.

18. Lay, A.J., et al., Phosphoglycerate kinase acts in tumour angiogenesis as a disulphide reductase. Nature, 2000. 408(6814): p. 869-73.

19. Ahmad, S.S., et al., Phosphoglycerate kinase 1 as a promoter of metastasis in colon cancer. Int J Oncol, 2013. 43(2): p. 586-90.

20. Carnielli, C.M., et al., Combining discovery and targeted proteomics reveals a prognostic signature in oral cancer. Nat Commun, 2018. 9(1): p. 3598.

21. Townsend, M.H., et al., Potential new biomarkers for endometrial cancer. Cancer Cell Int, 2019. 19: p. 19.

22. Fu, D., et al., PGK1 is a Potential Survival Biomarker and Invasion Promoter by Regulating the HIF-1aMediated Epithelial-Mesenchymal Transition Process in Breast Cancer. Cell Physiol Biochem, 2018. 51(5): p. 2434-2444.

23. Zhang, Y., et al., Activation of PGK1 under hypoxic conditions promotes glycolysis and increases stem cell-like properties and the epithelial-mesenchymal transition in oral squamous cell carcinoma cells via the AKT signalling pathway. Int J Oncol, 2020. 57(3): p. 743-755.

24. Zhang, W. and H.T. Liu, MAPK signal pathways in the regulation of cell proliferation in mammalian cells. Cell Res, 2002. 12(1): p. 9-18.

25. Robey, I.F., et al., Hypoxia-inducible factor-1alpha and the glycolytic phenotype in tumors. Neoplasia (New York, N.Y.), 2005. 7(4): p. 324-330.

26. Maxwell, P.H., et al., Hypoxia-inducible factor-1 modulates gene expression in solid tumors and influences both angiogenesis and tumor growth. Proc Natl Acad Sci U S A, 1997. 94(15): p. 8104-9.

27. Hay, N., Reprogramming glucose metabolism in cancer: can it be exploited for cancer therapy? Nature Reviews Cancer, 2016. 16(10): p. 635-649.

28. Patra, K.C., et al., Hexokinase 2 is required for tumor initiation and maintenance and its systemic deletion is therapeutic in mouse models of cancer. Cancer Cell, 2013. 24(2): p. 213-228.

29. Minchenko, O.H., et al., 6-Phosphofructo-2-kinase/fructose-2,6-bisphosphatase gene family overexpression in human lung tumor. Ukr Biokhim Zh (1999), 2005. 77(6): p. 46-50.

30. Xie, $\mathrm{H}$. , et al., Targeting lactate dehydrogenase-a inhibits tumorigenesis and tumor progression in mouse models of lung cancer and impacts tumor-initiating cells. Cell Metab, 2014. 19(5): p. 795-809. 
31. Cheng, J.Q., et al., Transforming activity and mitosis-related expression of the AKT2 oncogene: evidence suggesting a link between cell cycle regulation and oncogenesis. Oncogene, 1997. 14(23): p. 2793-801.

32. Mahajan, K. and N.P. Mahajan, PI3K-independent AKT activation in cancers: a treasure trove for novel therapeutics. Journal of cellular physiology, 2012. 227(9): p. 3178-3184.

33. Staal, S.P., Molecular cloning of the akt oncogene and its human homologues AKT1 and AKT2: amplification of AKT1 in a primary human gastric adenocarcinoma. Proceedings of the National Academy of Sciences, 1987. 84(14): p. 5034-5037.

34. Bellacosa, A., et al., Molecular alterations of the AKT2 oncogene in ovarian and breast carcinomas. International journal of cancer, 1995. 64(4): p. 280-285.

35. Cheng, J.Q., et al., Amplification of AKT2 in human pancreatic cells and inhibition of AKT2 expression and tumorigenicity by antisense RNA. Proceedings of the National Academy of Sciences, 1996. 93(8): p. 3636-3641.

36. Elstrom, R.L., et al., Akt stimulates aerobic glycolysis in cancer cells. Cancer Res, 2004. 64(11): p. 3892-9.

37. Hajduch, E., G.J. Litherland, and H.S. Hundal, Protein kinase B (PKB/Akt) - a key regulator of glucose transport? FEBS Letters, 2001. 492(3): p. 199-203.

38. Guo, Y.J., et al., ERK/MAPK signalling pathway and tumorigenesis. Exp Ther Med, 2020. 19(3): p. 1997-2007.

39. Pouysségur, J., F. Dayan, and N.M. Mazure, Hypoxia signalling in cancer and approaches to enforce tumour regression. Nature, 2006. 441(7092): p. 437-43.

40. Semenza, G.L. and G.L. Wang, A nuclear factor induced by hypoxia via de novo protein synthesis binds to the human erythropoietin gene enhancer at a site required for transcriptional activation. Mol Cell Biol, 1992. 12(12): p. 5447-54.

41. Muz, B., et al., The role of hypoxia in cancer progression, angiogenesis, metastasis, and resistance to therapy. Hypoxia (Auckland, N.Z.), 2015. 3: p. 83-92.

42. Giatromanolaki, A., et al., Relation of hypoxia inducible factor 1 a and $2 a$ in operable non-small cell lung cancer to angiogenic/molecular profile of tumours and survival. British journal of cancer, 2001. 85(6): p. 881-890.

43. Detterbeck, F.C., et al., The Eighth Edition Lung Cancer Stage Classification. Chest, 2017. 151(1): p. 193-203. 


\section{Figures}

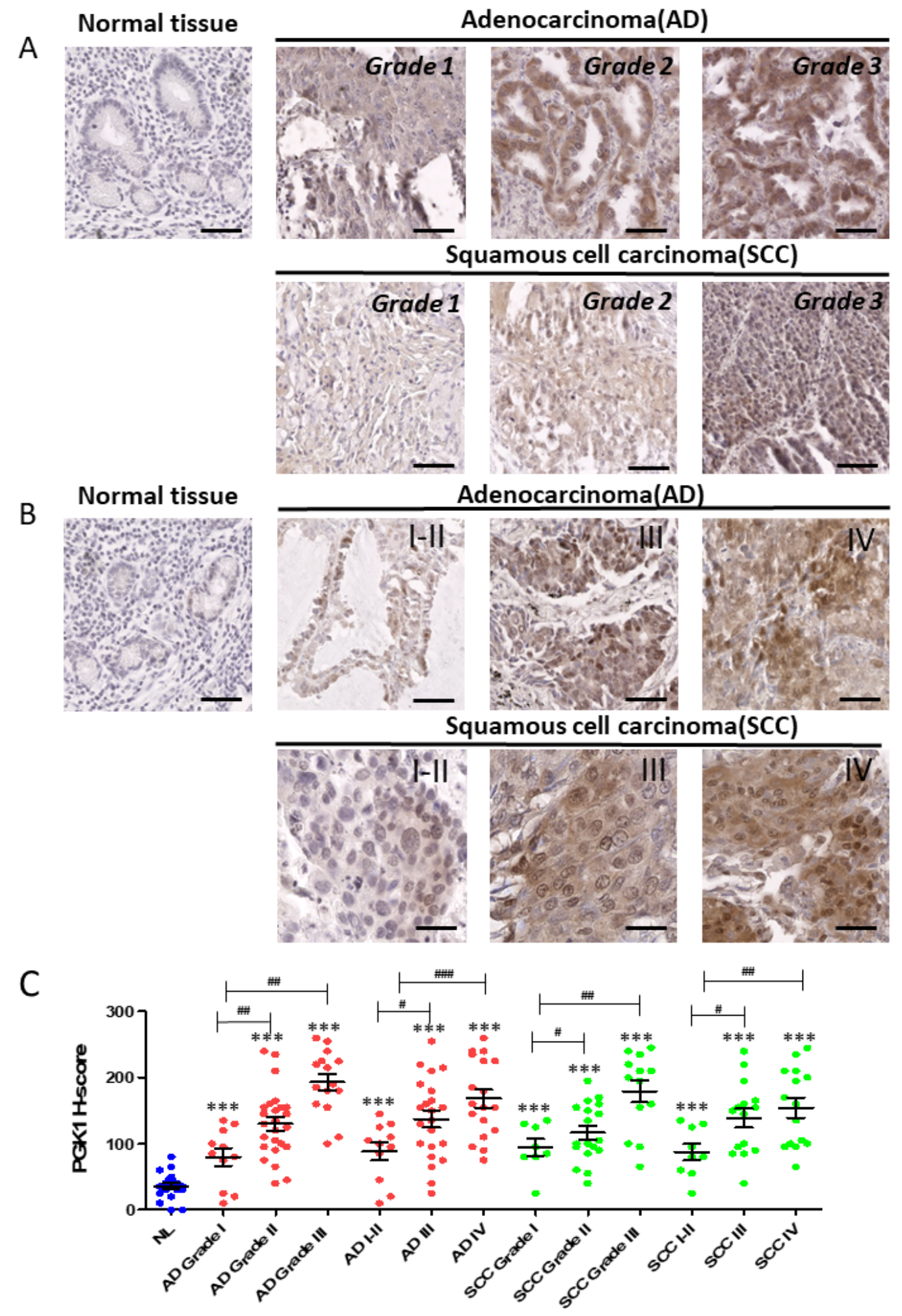

Figure 1

PGK1 expression level was upregulated in NSCLC patients. (A and B) IHC of representative patients' tissue samples from different types of lung tumors in different grades (A) and different stage (B) stained with anti-PGK1 antibody. Scale bars, $50 \mu \mathrm{m}$. (C) Relative staining intensity (H-score) for PGK1 in different 
grade and stage of different types of lung tumors. For adenocarcinoma (AD), $n=51$ ( $n=11$ grade 1, $n=25$ grade $2, n=15$ grade $3 ; n=11$ stage $I-I I, n=22$ stage III, $n=18$ stage IV). For squamous cell carcinoma (SCC) $n=29(n=8$ grade $1, n=18$ grade $2, n=13$ grade $3 ; n=9$ stage $I-I, n=15$ stage $I I I, n=15$ stage IV). For normal lung tissue $n=20$. Statistical analysis was performed by Student's $t$ test with two-tailed distribution. Data are shown as mean \pm SEM. $* \star \star, p<0.001$ versus normal lung tissue. $\#, p<0.05 ; \# \#, p<0.01 ; \# \# \#, p<0.001$. $\mathrm{NL}$, normal lung tissue.

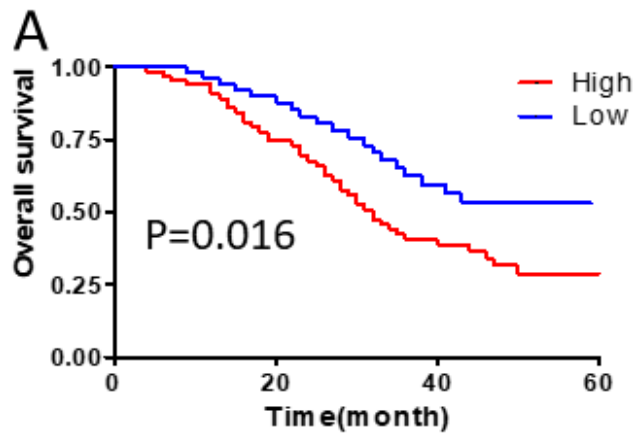

B

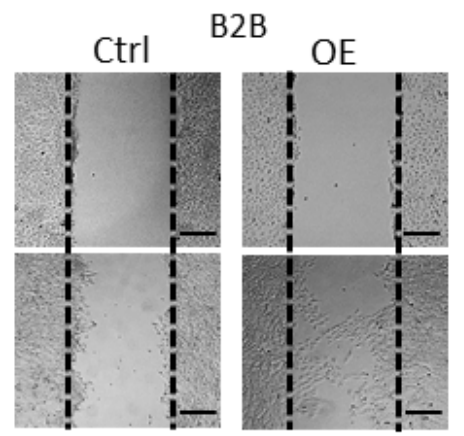

$$
\text { C }
$$

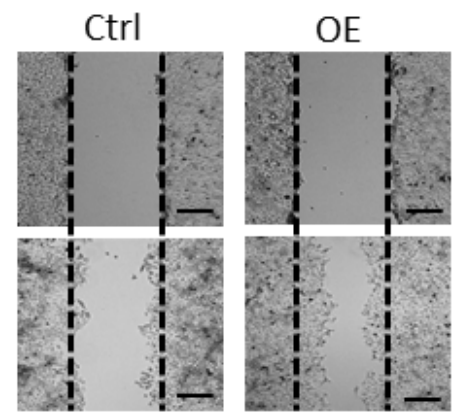

$\mathrm{E}$

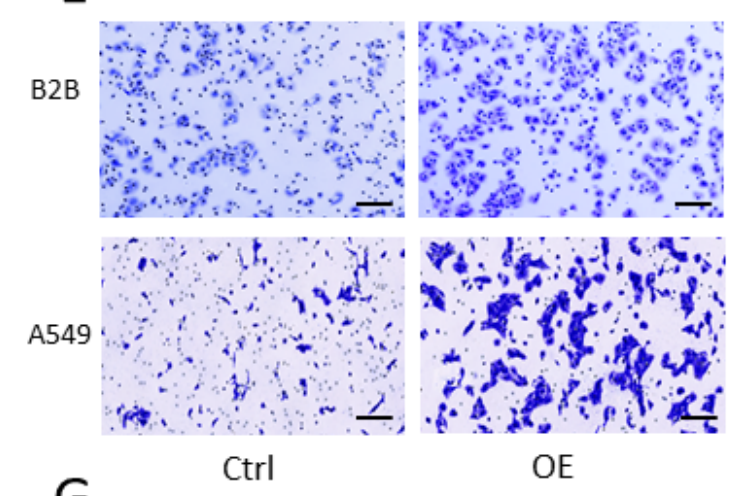

G Ctrl $\quad \mathrm{OE}$
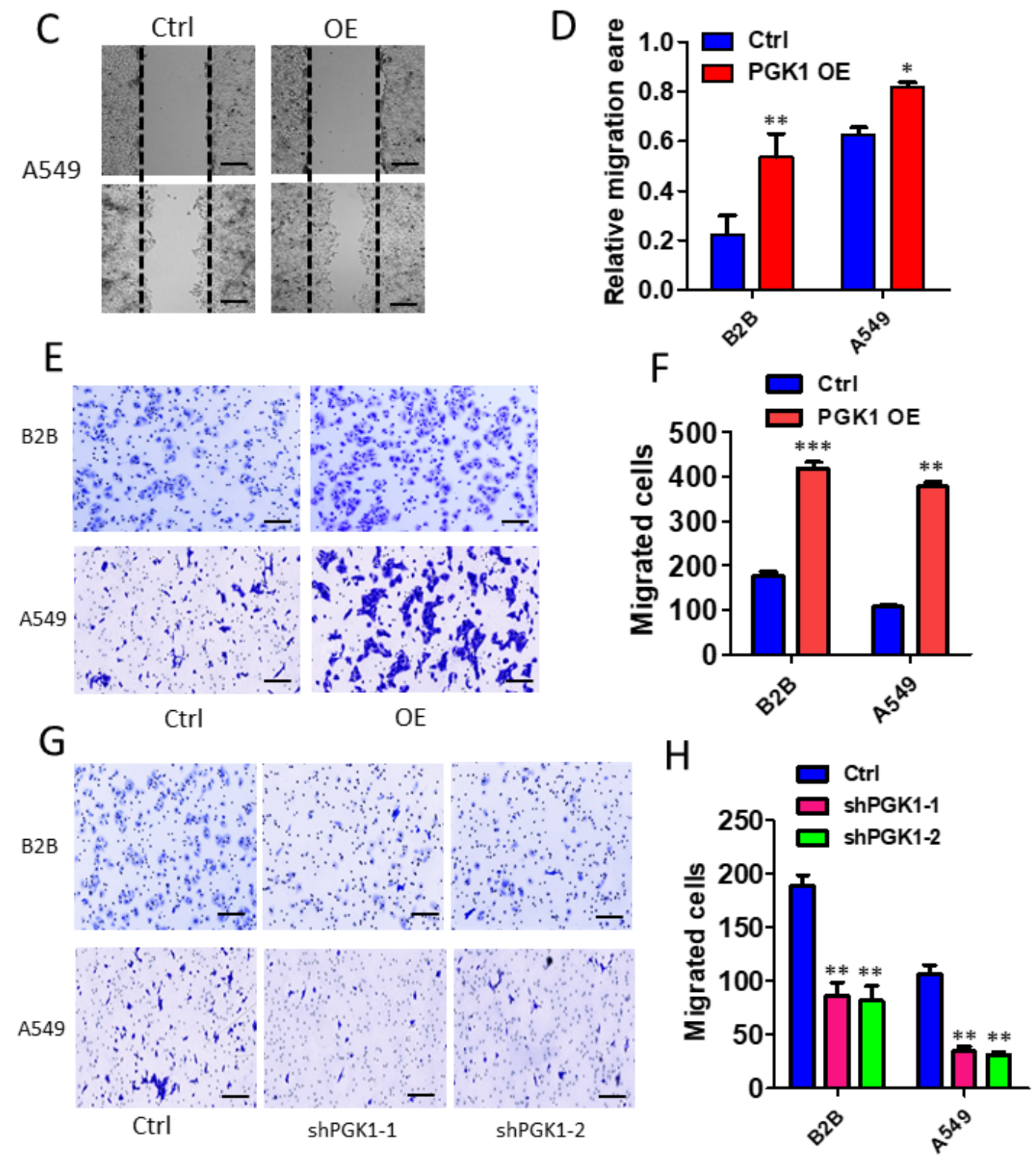

Figure 2 
PGK1 dysregulation affects cell migration. (A) Kaplan-Meier survival curves for overall survival in lung cancer patients. (B and C) Scratch wound healing assays with control and PGK1 OE B2B and A549 cells respectively. Scale bars, $100 \mu \mathrm{m}$. (D) Quantification of scratch wound healing assay. (E) Boyden chamber migration assay with control and PGK1 OE B2B and A549 cells. Scale bars, $100 \mu \mathrm{m}$. (F) Quantification of migrated cells in (E). (G) Boyden chamber migration assay with control and PGK1 silencing B2B and A549 cells. Scale bars, $100 \mu \mathrm{m}$. (H) Quantification of migrated cells in (G). All the data shown are mean \pm $\mathrm{SD}, *, p<0.05 ; * *, p<0.01 ; * * *, p<0.001$.

A

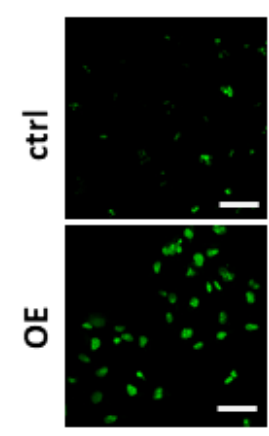

C

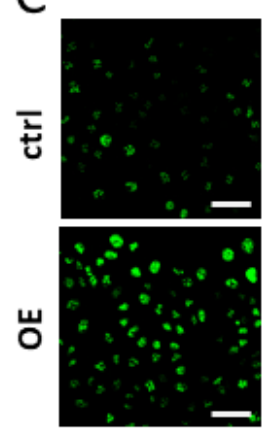

$\mathrm{E}$

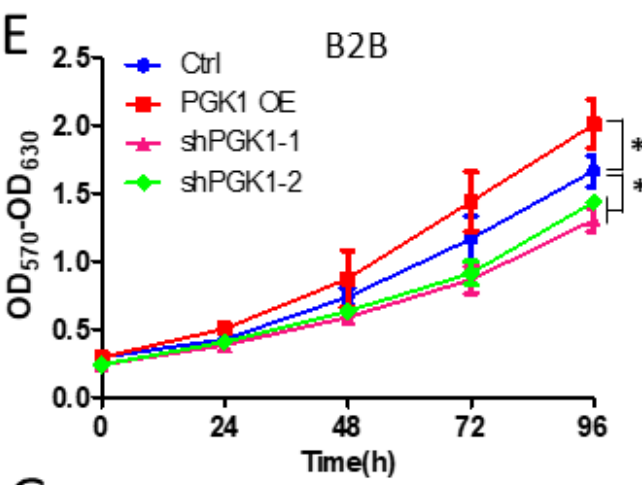

G
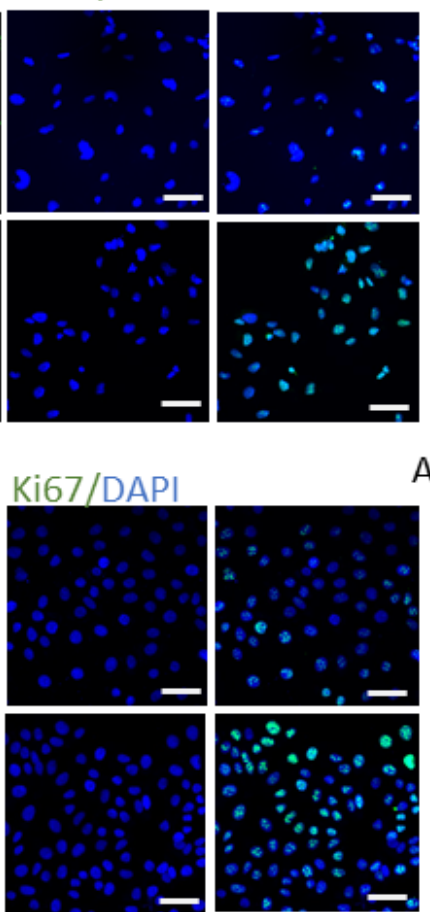

B2B

B

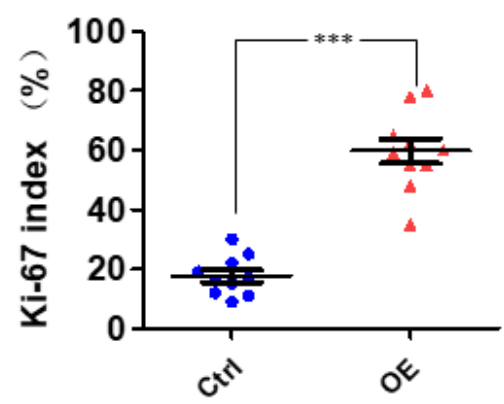

A549
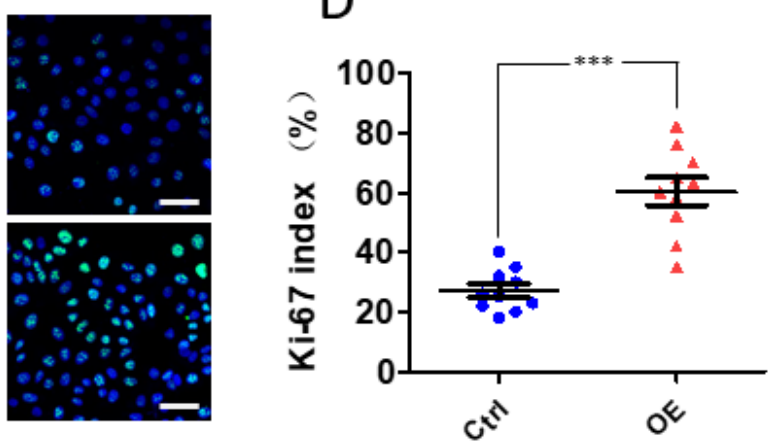

F
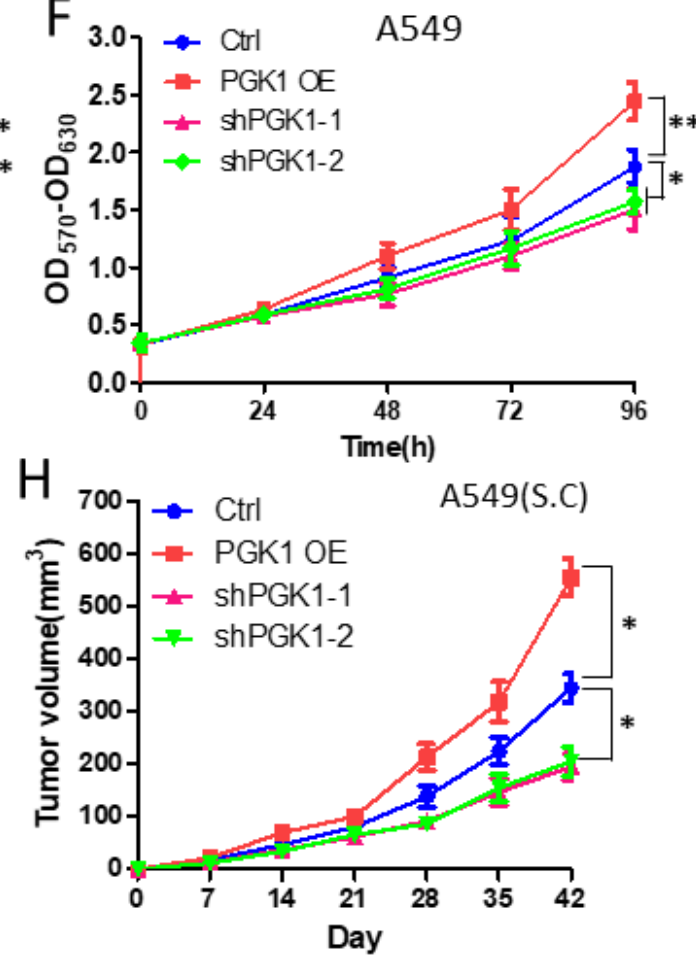


\section{Figure 3}

PGK1 promotes tumor growth both in vitro and in vivo. (A and C) Ki-67 immunofluorescence images of control and PGK1 OE B2B (A) and A549 (C) cells. Scale bar, $20 \mu \mathrm{m}$. (B and D) Ki-67 index in control and PGK1 OE B2B (B) and A549 (D) cells. Statistical analysis was performed by Student's t test with twotailed distribution. Data are mean \pm SEM. ${ }^{* \star *}, P<0.001$. (E and F) Cell viability of the control, PGK1 OE and PGK1 KD by two different shRNAs B2B (E) and A549 (F) cells was measured by MTT assay. Data are showed as mean + SD, *, p<0.05; **, p<0.01. (G) PGK1 control, PGK1 OE and PGK1 KD by two different shRNAs A549 cells were subcutaneously injected into nude mice. Mice were euthanized after 7weeks to harvest the xenograft tumors. Image of all xenograft tumors are shown. $(H)$ The tumor growth curves were plotted. Data are presented as mean $\pm S D(N=3) .{ }^{*}, p<0.05$ at day 42. 

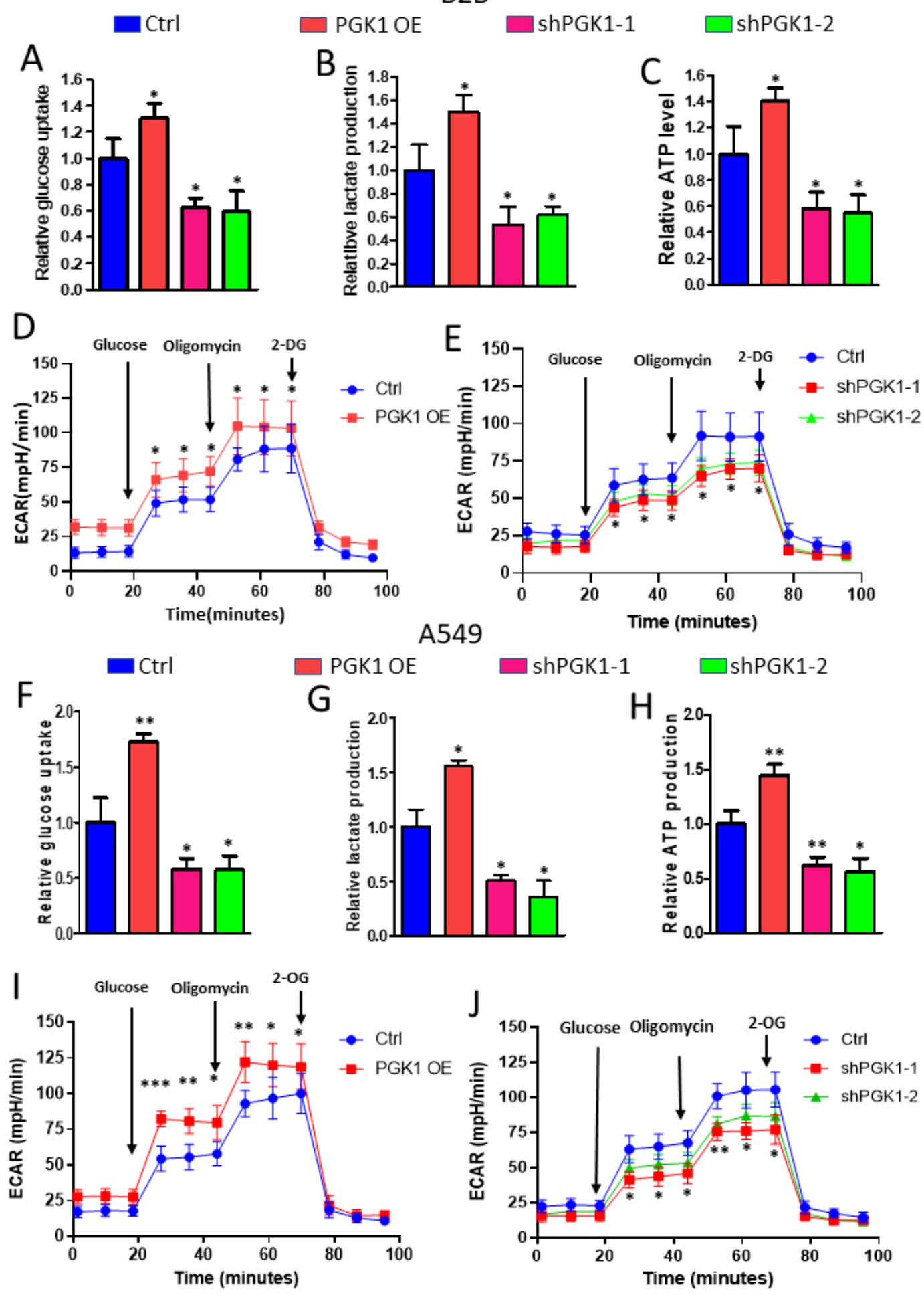

Figure 4

PGK1 plays a role in altering aerobic glycolysis in lung epithelia cells and NSCLC cells. (A-C) Glucose uptake, lactate secreted and ATP production of PGK1 control, PGK1-overexpression and PGK1 with shRNA-1 or shRNA-2 B2B cells were measured. Data shown as mean \pm SD of triplicate measurements that were repeated three times with similar results. *, $P<0.05$. ECAR assay for control and PGK1overexpression B2B cells (D) and ECAR assay for control, PGK1 with shRNA-1 or shRNA-2 B2B cells (E). 
Data were shown as mean \pm SD of quintuplicate measurements that were repeated three times with similar results. *, P<0.05. (F-H) Glucose uptake, lactate secreted and ATP production were measured again in PGK1 control, PGK1-overexpression and PGK1 with shRNA-1 or shRNA-2 A549 cells. Data shown as mean $\pm S D$ of triplicate measurements that were repeated three times with similar results. ${ }^{*}, P<0.05$; $\star *$, $P<0.01$. ( $I$ and $J$ ) ECAR assays were performed on control and PGK1-overexpression A549 cells ( $I$ ) and on control, PGK1 with shRNA-1 or shRNA-2 A549 cells $(\mathrm{J})$. Data were shown as mean \pm SD of quintuplicate measurements that were repeated three times with similar results. ${ }^{*}, P<0.05 ; \star \star, P<0.01 ; \star \star \star, P<0.001$.

B2B
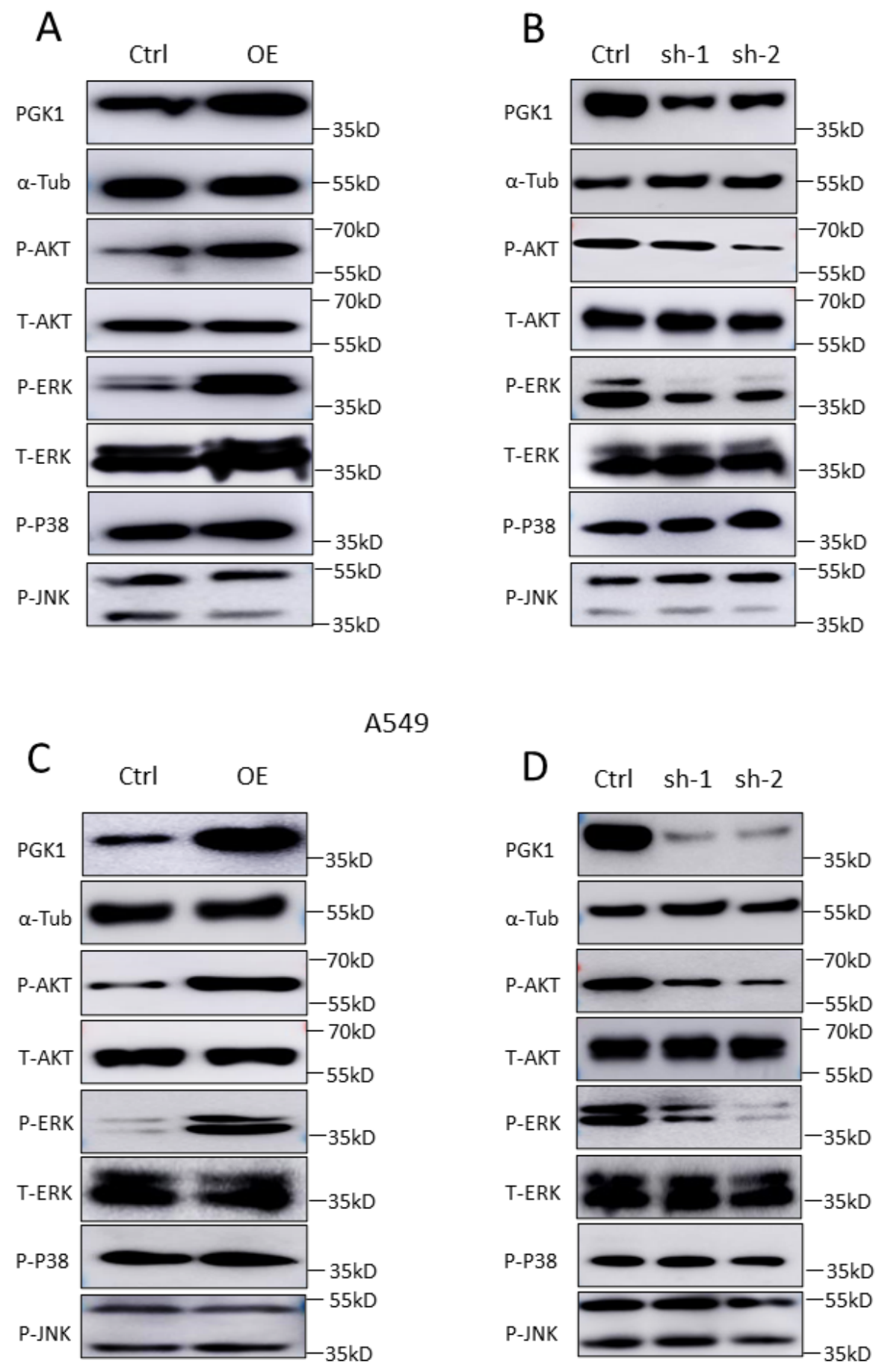

A549

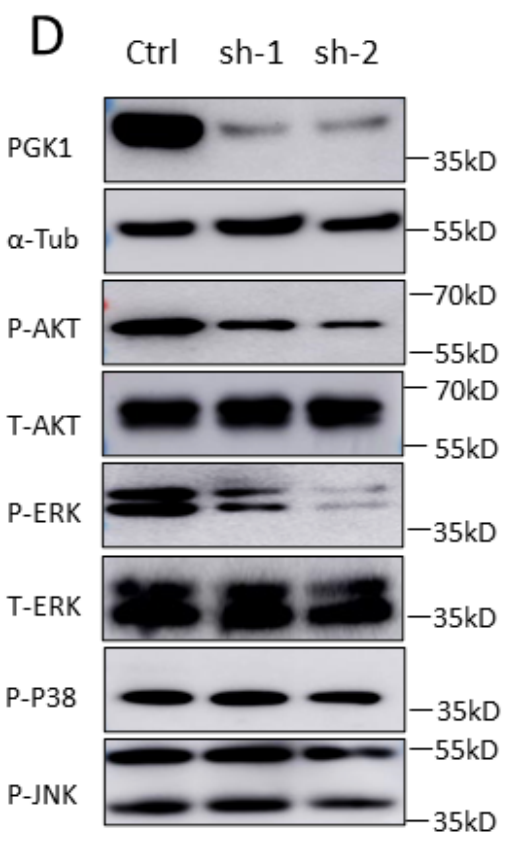


Figure 5

PGK1 promotes NSCLC progression by activating AKT/ERK signaling pathway. (A) Western blot analysis for activated AKT, P38, JNK, ERK of control and PGK1-overexpression B2B cells. (B) Western blot analysis for activated AKT, P38, JNK, ERK of control and PGK1 with shRNA-1 or shRNA-2 B2B cells. (C) Western blot analysis for activated AKT, P38, JNK, and ERK of control and PGK1-overexpression A549 cells. (D) Western blot analysis for activated AKT, P38, JNK, ERK of control and PGK1 with shRNA-1 or shRNA-2 A549cells. a-tub, a-tubulin.

A

Hypoxia $\quad 0 \mathrm{~h} 8 \mathrm{~h} 24 \mathrm{~h} 48 \mathrm{~h}$
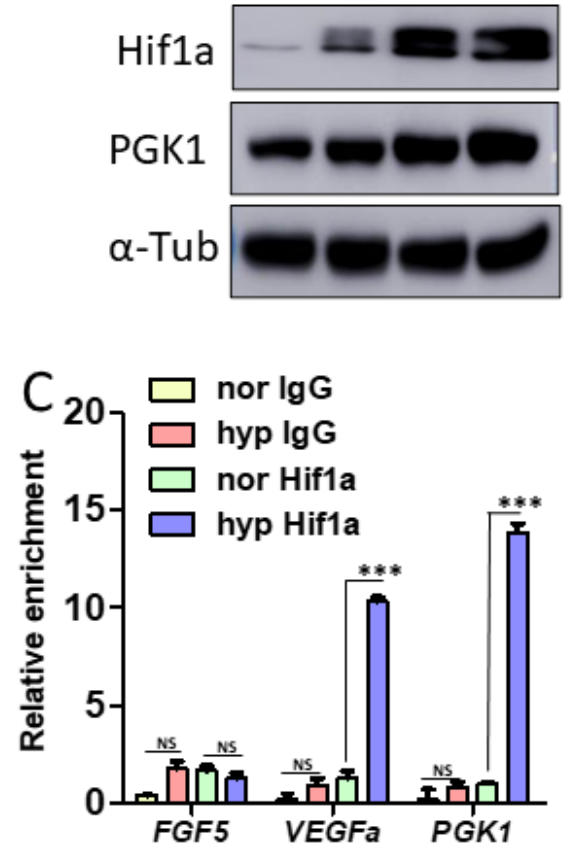

$E$

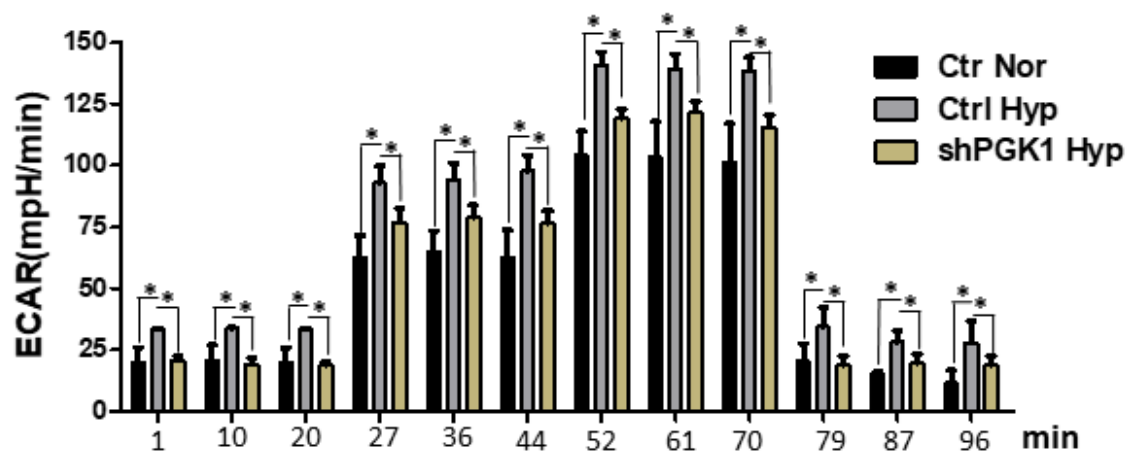

- Ctrl Nor

$\mathrm{F}$

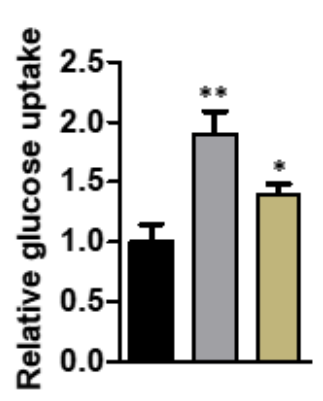

B
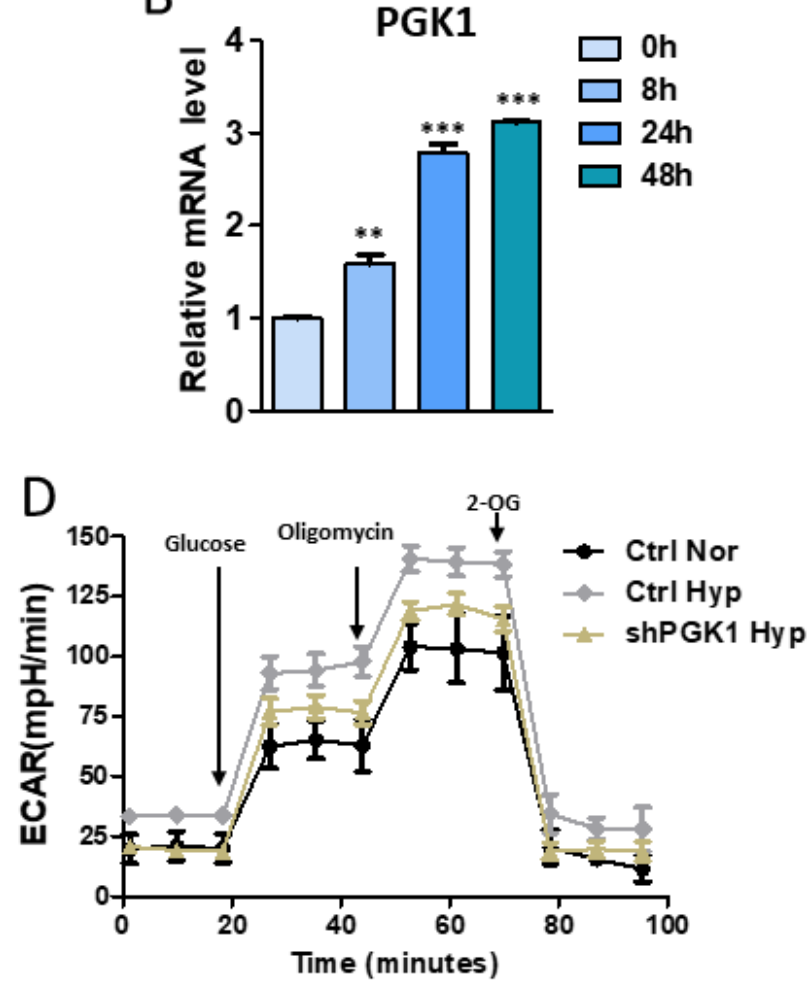

Ctrl Hyp

$\square$ shPGK1 Hyp

G
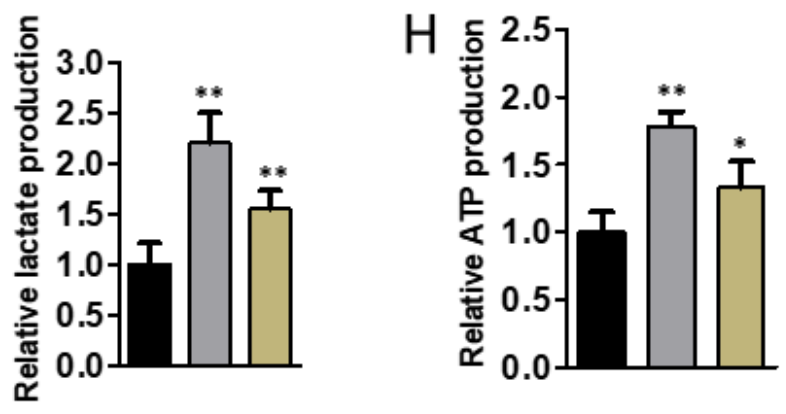


\section{Figure 6}

PGK1 is HIF1a target gene and plays an essential role in hypoxia-induced shift of glycolysis pathway. (A) Western bolt analysis of HIF1a and PGK1 in different time points of A549 cells treated in hypoxia. (B) qPCR analysis of PGK1 expression in A549 cells treated in hypoxia for different time points. Data shown are mean $\pm S D$ of three independent experiments. $* \star, P<0.01$; $* \star \star, P<0.001$. (C) Chip-qPCR analysis of IgG and HIF1 a occupancy at VEGFa and PGK1 promoter and FGF5 3' UTR region. Data shown are mean \pm SD of two independent experiments. NS, no significance. $* \star *, P<0.001$. ( $D$ and $E$ ) ECAR assays were performed on PGK1 control A549 cells treated in normoxia or hypoxia and PGK1-overexpression A549 cells treated in hypoxia. Data were shown as mean \pm SD of quintuplicate measurements that were repeated three times with similar results. *, P<0.05. (F-H) Glucose uptake, lactate secreted and ATP production of PGK1 control treated in normoxia or hypoxia and PGK1-overexpression A549 cells were measured. Data shown as mean $\pm S D$ of triplicate measurements that were repeated three times with similar results. *, $\mathrm{P}<0.05 ;$ **, $\mathrm{P}<0.01$. Nor, normoxia; Hyp, hypoxia. 

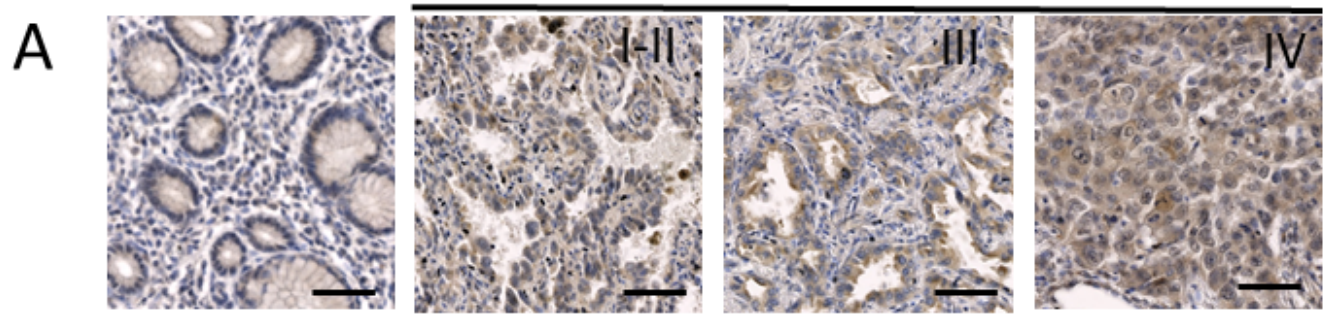

Squamous cell carcinoma(SCC)
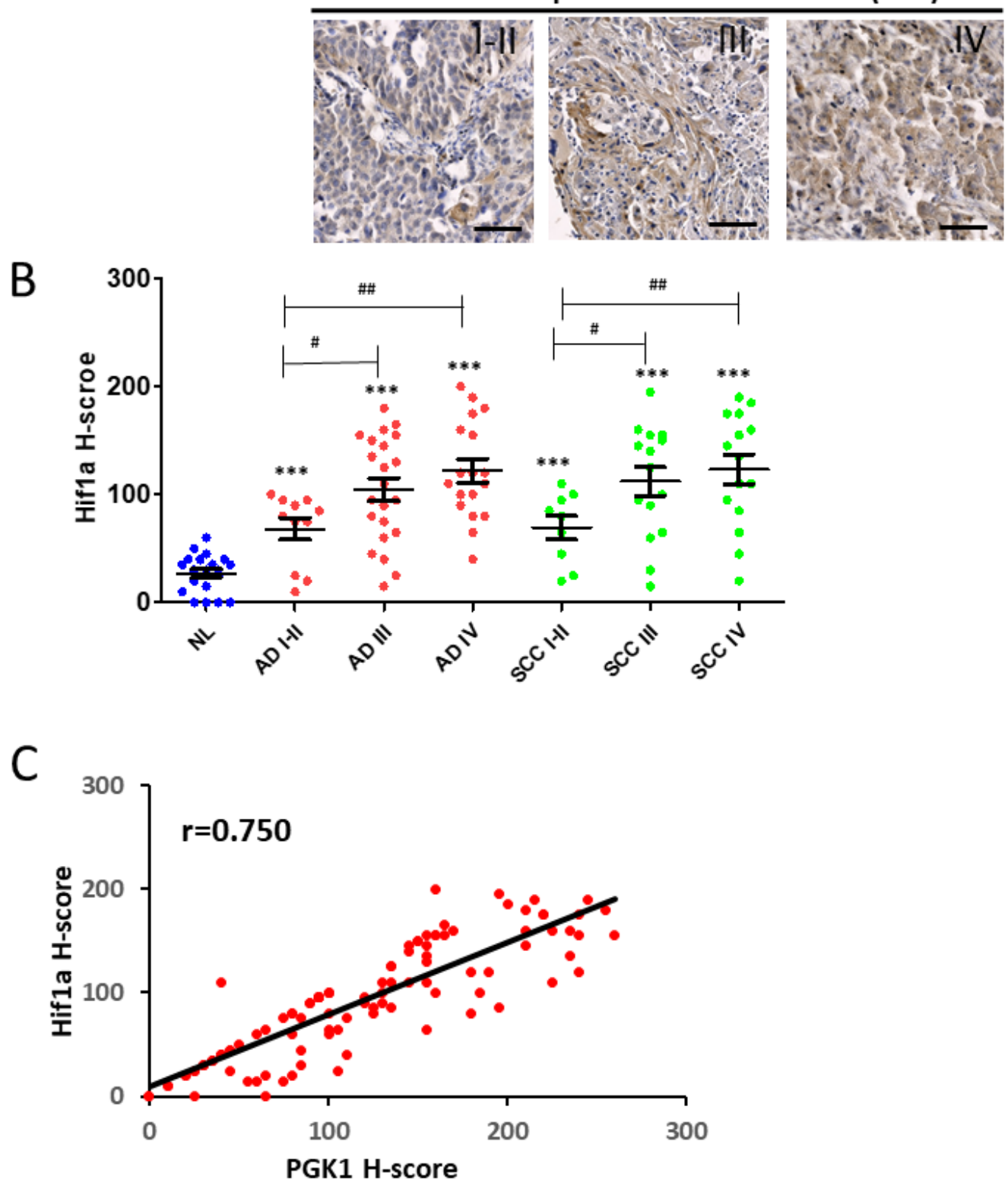

Figure 7

HIF1 a levels positively correlate with PGK1 levels in lung cancer patients. (A) IHC of representative patients' tissue samples from different types of lung tumors in different stage stained with anti-HIF1a antibody. Scale bars, $50 \mu \mathrm{m}$. (C) Relative staining intensity (H-score) for HIF1a in different stage of different types of lung tumors. For adenocarcinoma (AD), $n=51$ ( $n=11$ stage I-II, $n=22$ stage III, $n=18$ stage IV). For squamous cell carcinoma (SCC) $n=29$ ( $n=9$ stage I-II, $n=15$ stage III, $n=15$ stage IV). For normal 
lung tissue $n=20$. Statistical analysis was performed by Student's $t$ test with two-tailed distribution. Data are shown as mean \pm SEM. $* \star \star, p<0.001$ versus normal lung tissue. $\#, p<0.05 ; \# \#, p<0.01$. (D) Correlation between the relative staining intensity for PGK1 and HIF1a; r, Pearson correlation coefficient. NL, normal lung tissue.

\section{Supplementary Files}

This is a list of supplementary files associated with this preprint. Click to download.

- TableS1.docx

- TableS2.docx 